\section{Systemic Infection due to Acremonium after Allogeneic Peripheral Blood Stem Cell Transplantation}

\author{
Rie Yamazaki, Takehiko Mori, Yoshinobu Aisa, \\ Tomonori Nakazato, Ai Mihara, Yasuo Ikeda and \\ Shinichiro Okamoto
}

Key words: Acremonium, micafungin, 1,3-beta-D-glucan, myelodysplastic syndrome, stem cell transplantation

\section{(DOI: 10.2169/internalmedicine.45.1827)}

Acremonium, formerly known as Cephalosporium, is ubiquitously present in soil, insects, plants, and other environmental substrates (1). In rare cases, Acremonium has caused local infection in humans. Disseminated infections are even more rare, with the reported cases being limited to immunocompromised patients (1). Here, we report a patient with myelodysplastic syndrome (MDS) with profound neutropenia who developed disseminated Acremonium infection during treatment with micafungin.

A 34-year-old woman with MDS underwent allogeneic cord blood transplantation from an unrelated donor after being conditioned with total body irradiation and high-dose cytarabine. The graft was rejected, and then she underwent allogeneic peripheral blood stem cell transplantation from her mother. She was conditioned with cyclophosphamide, fludarabine, and anti-thymocyte globulin. Graft engraftment was achieved. Around day 35, pancytopenia developed, and the relapse of MDS was diagnosed. She remained severely neutropenic and received high-dose glucocorticoid therapy for severe graft-versus-host disease (GVHD). On day 100 post-transplant, when she had been on fluconazole for 4 months, a chest roentgenogram revealed pulmonary infiltrates on the left upper lobe with an increased serum level of beta-D-glucan. Fluconazole was discontinued, and micafungin was initiated at a daily dose of $300 \mathrm{mg}$. In spite of the therapy with micafungin, her pulmonary infiltrates extended. Multiple painful erythematous papular skin lesions developed on her extremities and back. The culture of a specimen obtained from these papules and blood grew Acremonium species. She died due to respiratory failure. The minimum inhibitory concentration (MIC) of micafungin against an isolate was later examined, and was found to be $0.125 \mu \mathrm{g} / \mathrm{ml}$.

In this case, pneumonia due to Acremonium progressed to disseminated fungemia and multiple skin lesions even after the prompt initiation of micafungin, indicating that micafungin was clinically ineffective against Acremonium. Previous studies evaluating the activity of anti-fungal agents against Acremonium both in vitro and in vivo indicated that amphotericin B, itraconazole, and voriconazole are promising agents for the treatment of Acremonium infection $(1,2)$. Micafungin is a compound of the echinocandin class that acts by inhibiting the production of 1,3-beta-D-glucan, a key component in fungal cell wall synthesis in molds and yeasts. However, investigation into the activity of echinocandins against Acremonium has been limited to two previous reports, in which these compounds were shown to exert limited activity against Acremonium strictum $(3,4)$. Indeed, micafungin was clinically ineffective against Acremonium infection in the present patient with a markedly elevated serum level of 1,3-beta-D-glucan, and the MIC of micafungin against the isolate was higher than those of Aspergillus species (0.008-0.017 $\mu \mathrm{g} / \mathrm{ml})(5)$.

In conclusion, physicians should be aware that Acremonium is one of the causative fungi of systemic infection in severely immunocompromised patients who have been on either fluconazole or micafungin. 


\section{References}

1. Guarro J, Gams W, Pujol I, Gene J. Acremonium species: New emerging fungal opportunists-in vitro antifungal susceptibilities and review. Clin Infect Dis 25: 1222-1229, 1997.

2. Wildfeuer A, Seidl HP, Paule I, Haberreiter A. In vitro evaluation of voriconazole against clinical isolates of yeast, moulds and dermatophytes in comparison with itraconazole, ketoconazole, amphotericin B and griseofulvin. Mycoses 41: 309-319, 1998.

3. Esplinel-Ingroff A. Comparison of in vitro activities of new triazole SCH56592 and the echinocangins MK-0991 (L-743, 872) and
LY303366 against opportunistic filamentous and dimorphic fungi and yeasts. J Clin Microbiol 36: 2950-2956, 1998.

4. Odabashi Z, Paetznick VL, Rodriguez JR, Chen E, OstroskyZeichner L. In vitro activity of anidulafungin against selected clinically important mold isolates. Antimicrob Agents Chemother 48: 1912-1915, 2004.

5. Ikeda $F$, Otomo $K$, Nakai $T$, et al. In vitro activity of a new lipopeptide antifungal agent, micafungin against a variety of important fungi. Jpn J Chemother 50 (S-1): 8-19, 2002.

(C) 2006 The Japanese Society of Internal Medicine http://www.naika.or.jp/imindex.html 